\title{
Experimental study of metastable liquids decay during depressurization in cylindrical channel
}

\author{
Igor Kozulin ${ }^{1,2, *}$, German Bartkus ${ }^{1,2}$, and Sergey Dimov ${ }^{1}$ \\ ${ }^{1}$ SB RAS, Institute of Thermophysics, 630090 pr. Akademika Lavrentjeva 1, Novosibirsk, Russia \\ ${ }^{2}$ Novosibirsk State University, 630090 st. Pirogova 1, Novosibirsk, Russia
}

\begin{abstract}
Patterns of the nonequilibrium phase transitions during propagation of an adiabatic wave phase transition in metastable refrigerants R141b, R113 and pentane $\mathrm{C}_{5} \mathrm{H}_{12}$ were experimentally established. Experiments were carried out in a wide range of initial temperatures and pressures in a transparent cylindrical channel. Visualization of liquids decay was performed using high-speed video camera.
\end{abstract}

\section{Introduction}

A research of fluid destruction at high specific energy impacts is necessary for development of the nonequilibrium phase transitions theory, as well as for applications related to developing the security systems, the energy systems, and solving the security problems during storage of liquefied gases.

A distribution of phase transition at depressurization of the cylindrical channel was studied in [1]. The phase transition in channels with different shapes was studied in the paper [2] and it was shown that the wave speed is independent from the channel diameter and its shape. This paper continues the experimental study of [3] and [4]. In these articles, the adiabatic evaporation waves were investigated during the rapid transition of Freon in the metastable state after pressure relief. An analysis of the published studies shows that the phase transition in the metastable liquid was not completely studied. A liquid vaporization mechanism was not defined on the wave front. The aim of this work is to determine the patterns of surface decay during depressurization of metastable Freon R141b, R113 and pentane $\mathrm{C}_{5} \mathrm{H}_{12}$. The fluids selection is caused by a significant difference in their thermal properties, including vapor density, liquid heat capacity and latent heat of vaporization. A phase transition visualization was performed using a high-speed video camera.

\section{Experimental setup}

The study of propagation of wave phase transition in metastable liquid was made using the experimental setup, Fig. 1.

\footnotetext{
*Corresponding author: igornt@yandex.ru
} 


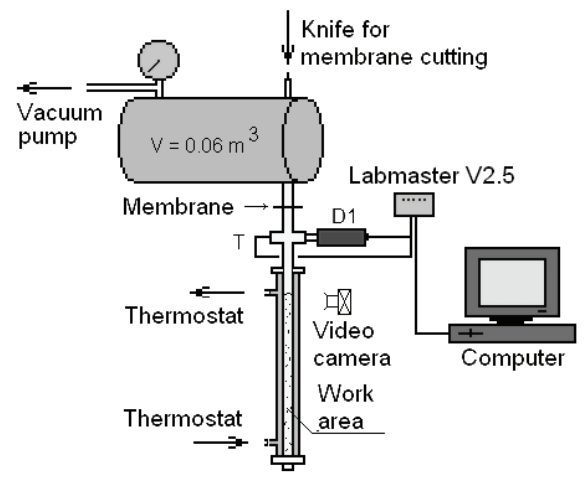

Fig. 1. The experimental setup.

The experimental setup includes a low-pressure chamber volume of 60 liters and a test section with a superheated liquid separated from chamber by a membrane. The membrane was cut by a special knife at a specified time. Refrigerants R141b, R113 and pentane $\mathrm{C}_{5} \mathrm{H}_{12}$ were used as working fluids. The cylindrical glass channel was used as a test section. The inner diameter is $16 \mathrm{~mm}$, the cannel length is $300 \mathrm{~mm}$. The test section is located vertically and it has a thermostatic jacket in which water is pumped from the thermostat. The thermostat heated the test section to a determined temperature $\mathrm{T}_{0}$.

The originality of this set-up is that that the test section is made from glass. It allows us to visualize the boiling liquid and study the detailed structure of the adiabatic wave front in the superheated liquid. The initial temperature range was varied from 35 to $80{ }^{\circ} \mathrm{C}$ and measured with an accuracy of $0.3{ }^{\circ} \mathrm{C}$. Prior to the experiment in a low-pressure chamber with vacuum, the pump created a vacuum in a low-pressure chamber measured with an accuracy of 0.005 bar. The initial pressure range produced in the low-pressure chamber was $\mathrm{P}_{\text {res }}=0.04-0.07$ bar. The pressure sensor DMP 330L with a measuring range from 0 to 16 bar is located at the top of the test section. The copper - constantan thermocouple was mounted at the top of the test section. During the experiment, the data from sensors handled from ADC Labmaster V2.5 in PC. Using high-speed video camera Optronis CR600x2, the registration of metastable liquids decay was made.

\section{Results and discussion}

The adiabatic phase transition initiated by the pressure relief was caused by the membrane rupture. The bubbles' microbursts are triggered on the surface layer of the superheated liquid. The metastable liquid formed pulsating waves of intense phase transition with the cellular structure interphase border after the establishment of pressure.

Fig. 2 (a) shows the dynamics of boiling refrigerant R141b at the initial temperature and pressure: $\mathrm{T}_{0}=35.4{ }^{\circ} \mathrm{C}, \mathrm{P}_{\text {res }}=0.04$ bar. A video of the phase transition was carried out using a high-speed video camera (500 fps) with transmitted light. It is seen from the photos that there are the end stage of emission and stop boundaries $(\mathrm{t}=0 \mathrm{~ms})$ and the stage of intensive evaporation and moving the boundaries $(\mathrm{t}=6 \mathrm{~ms})$ of supersaturated vapor. This process is periodic; as a result of which, it is possible to observe the development of the interfacial waves of surface boiling. Velocity of boiling refrigerant R141b is shown in Fig. 2 (b) at initial temperature $\mathrm{T}_{0}=35.4{ }^{\circ} \mathrm{C}$.

The local interface velocity of boiling was determined with the help of a video image processing program which allows determining the coordinates of the current boiling front position along the entire border between the two phases. These coordinates are averaged to obtain the current boiling front position for a given image. 


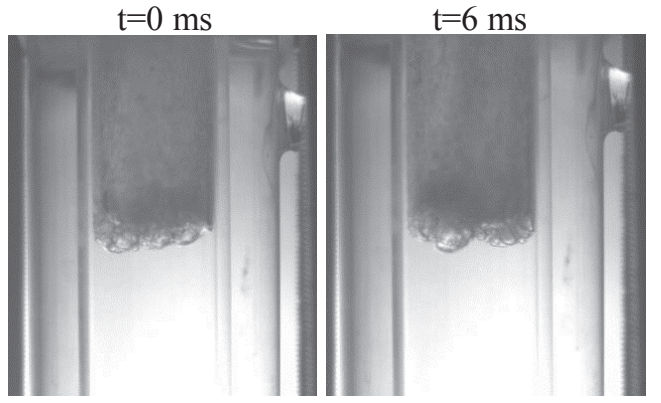

(a)

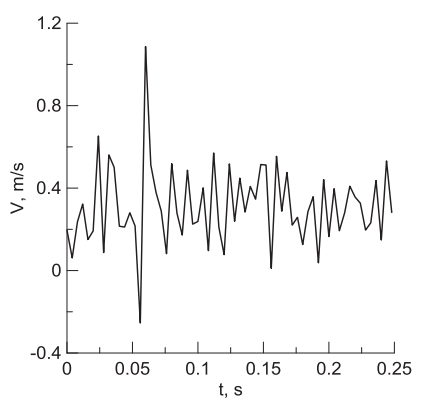

(b)

Fig. 2. The dynamics of refrigerant $\mathrm{R} 141 \mathrm{~b}$ boiling at initial temperature and pressure: $\mathrm{T}_{0}=35.4{ }^{\circ} \mathrm{C}$, $P_{\text {res }}=0.04$ bar. (a) Visualization at $500 \mathrm{fps}$. (b) Velocity of boiling, depending from the time.

The average speed of the boiling front at $\mathrm{T}_{0}=35.4{ }^{\circ} \mathrm{C}$ for refrigerant $\mathrm{R} 141 \mathrm{~b}$ was $0.31 \mathrm{~m} / \mathrm{s}$. There are significant pulsations of velocities while moving an interphase surface. These pulsations are greatly exceeded its mean value. The stage of active vaporization and moving of the front changes to the stage of stopping and collapsing the front, but the average speed of the wave is constant during the whole phase transition process. The average velocity of the wave depends slightly on the pressure in the low pressure chamber and is determined by initial temperature of the liquid.

The experimental study of the metastable decay of refrigerant R113 was performed. Fig. 3 (a) shows the dynamic of the surface phase transition for refrigerant R113 at temperature and pressure: $\mathrm{T}_{0}=65.2{ }^{\circ} \mathrm{C}, \mathrm{P}_{\text {res }}=0.036$ bar at boiling nucleation in a surface layer. The figure shows that the evaporation front moves from top to bottom, the boiling is accompanied by high-intensity fluctuations at the interface surface between the liquid and gas phases. Fig. 3 (b) shows the velocity of interface surface of boiling refrigerant R113 at temperature $\mathrm{T}_{0}=65.2{ }^{\circ} \mathrm{C}$. The average speed of boiling front is $0.32 \mathrm{~m} / \mathrm{s}$.

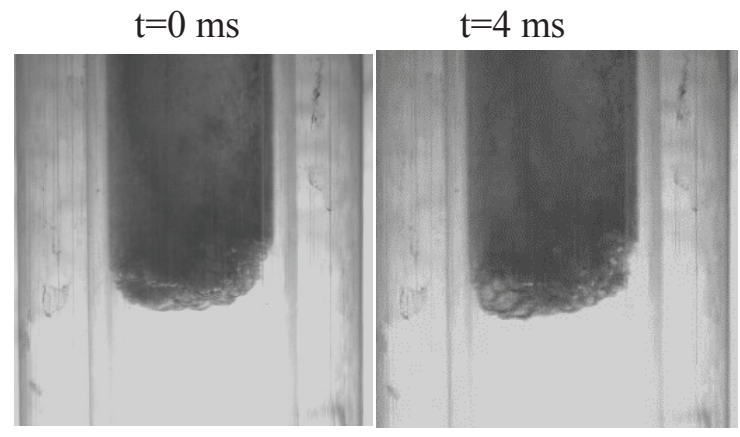

(a)

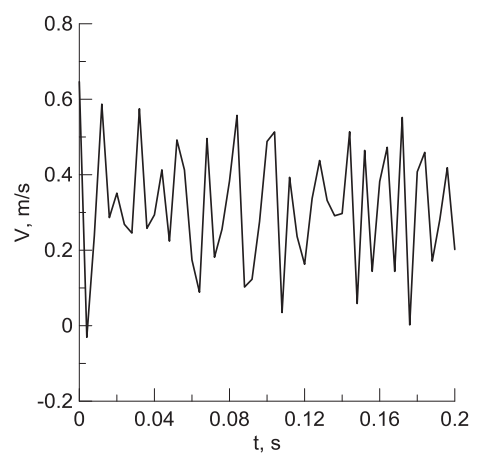

(b)

Fig. 3. The dynamics of refrigerant R113 boiling at initial temperature and pressure: $\mathrm{T}_{0}=65.2{ }^{\circ} \mathrm{C}$, $\mathrm{P}_{\text {res }}=0.036$ bar. (a) Visualization at 500 fps. (b) Velocity of boiling, depending from the time.

The experimental study of the metastable decay of pentane was performed. Fig. 4 (a) shows the dynamic of the surface phase transition for refrigerant R113 at temperature and pressure: $\mathrm{T}_{0}=48.55{ }^{\circ} \mathrm{C}, \mathrm{P}_{\text {res }}=0.04$ bar at boiling nucleation in a surface layer. Fig. 4 (b) shows velocity of the pentane wave boiling at temperature $\mathrm{T}_{0}=48.55{ }^{\circ} \mathrm{C}$. The average speed of boiling is $0.52 \mathrm{~m} / \mathrm{s}$. 


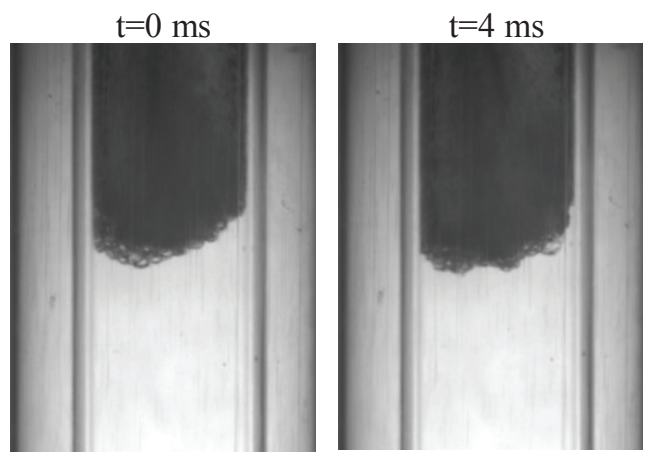

(a)

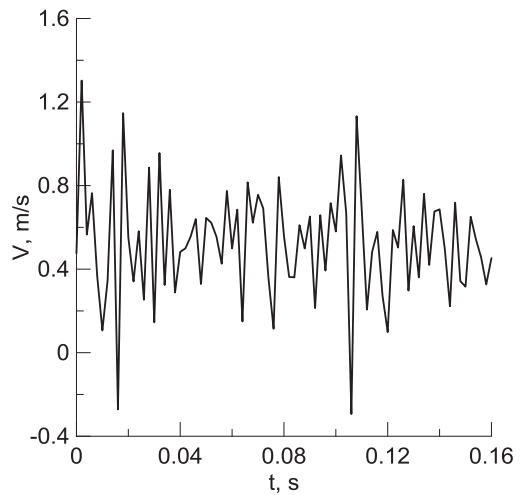

(b)

Fig. 4. The dynamics of pentane boiling at initial temperature and pressure: $\mathrm{T}_{0}=48.55^{\circ} \mathrm{C}$, $P_{\text {res }}=0.04$ bar. (a) Visualization at 500 fps. (b) Velocity of boiling, depending from the time.

A visualization of the first nucleation wave on the interfacial meniscus was made for pentane with the help of video camera Optronis CR600x2. The camera settings were $15000 \mathrm{fps}$ and the picture resolution $128 \times 128$ px, Fig. 5 .

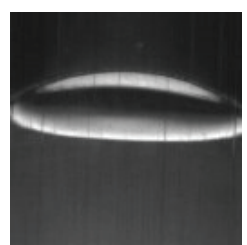

$\mathrm{t}=0 \mu \mathrm{s}$

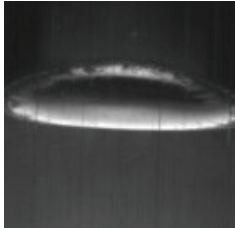

$\mathrm{t}=67 \mu \mathrm{s}$

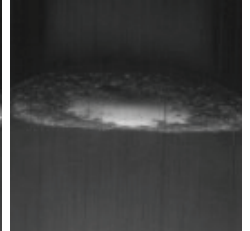

$\mathrm{t}=200 \mu \mathrm{s}$

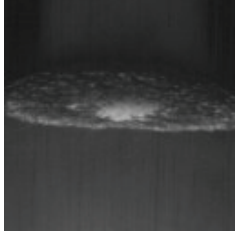

$\mathrm{t}=267 \mu \mathrm{s}$

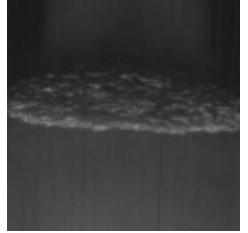

$\mathrm{t}=400 \mu \mathrm{s}$

Fig. 5. The initial stage of a phase transition of pentane at temperature and pressure: $\mathrm{T}_{0}=50.07{ }^{\circ} \mathrm{C}$, $\mathrm{P}_{\text {res }}=0.05$ bar. Video frame rate $-15000 \mathrm{fps}$.

Figure 5 shows the nucleation of the liquid boiling centers initiated on the surface layer in the meniscus interfacial region. The propagation of the wave nucleation of boiling centers is most often from the channel walls to its center. The bubble's growth is triggered by the rupture of the membrane on the surface layer of the superheated liquid. Then the formation of liquid pulsating decay comes with the cellular structure in the inter phase surface. The wave begins on the fluid surface layer near the wall in the area where the work for generation of critical bubble is reduced.

Fig. 6 (a) shows the average speed of the interphase boundary depending on the initial temperature during boiling Freon R141b, R 113 and pentane. The graph shows that at the same heating rate the velocity of interface motion for pentane is greater than the average velocity of the inter phase boundary for Freon. The average speed of the initial stage of the phase transition for pentane was determined, Fig 6 (b). Figure 6 shows that the rate of initial wave formation at the interface surface is significantly greater than the rate of liquid evaporation during the developed boiling. 


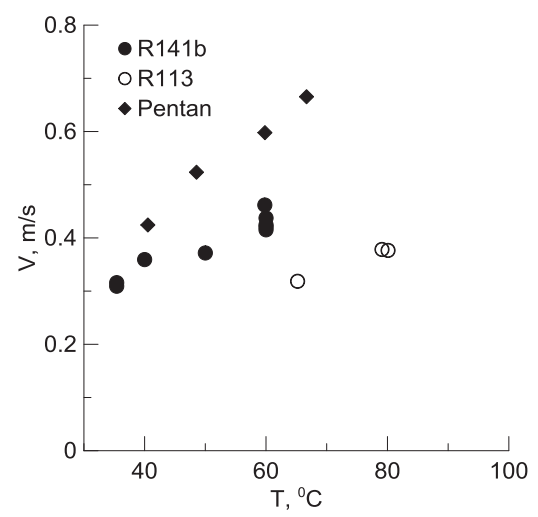

(a)

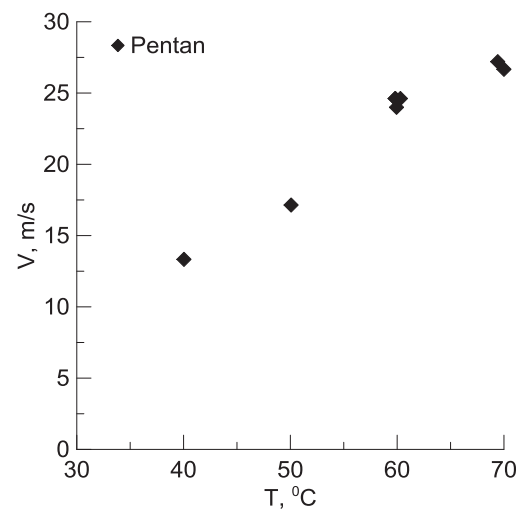

(b)

Fig. 6. (a) The average speed of the interphase boundary during boiling depending on the initial temperature; (b) The average speed of the initial stage of the phase transition for pentane depending on the initial temperature.

\section{Conclusion}

The experimental studies were made for investigation of the adiabatic wave evaporation with rapid transition refrigerant R141b, R113, pentane in a metastable state by the pressure reduction. It was found that the phase transition in the metastable liquid passes under development of multiscale turbulence in the liquid and vapor phases. Using the high-speed video camera, the images of surface phase transition were taken, the pulsating character was established. The rate of formation of the wave phase transition for the metastable pentane was established. Velocity pulsations of the phase transition were determined for the metastable pentane and Freon during the boiling depending on the initial temperature difference.

The study was performed in the Kutateladze Institute of Thermophysics SB RAS and was supported by grant RFBR No. 15-08-06870 A.

\section{References}

1. V.V. Kuznetsov, I.A. Kozulin, O.V. Vitovsky, J. Eng.Therm. 21, 136-143 (2012)

2. P. Reinke, G. Yadigaroglu, Int. J. of Multiphase Flow. 27, 1487-1516 (2001)

3. L.G. Hill, B. Sturtevant, Int. Union of Theor. and Applied Mechanics, 25-37 (1990)

4. P. Reinke, G. Yadigaroglu, Int. J. of Multiphase Flow. 27, 1487-1516 (2001) 